\title{
Can 6-minute walk test predict severity of obstructive sleep apnea syndrome?
}

Parisa Adimi Naghan', Oldooz Aloosh ${ }^{2 *}$, Hamze Ali Torang ${ }^{3}$ and Majid Malekmohammad ${ }^{3}$

\begin{abstract}
Background: When considering the benefits of the 6-min walking test (6-MWT) in research fields and the need of treatment for moderate to severe obstructive sleep apnea (OSA) patients, research in this field is of great advantage and may have a significant role in therapeutic grounds.

Methods: This cross-sectional study was conducted on 47 patients with confirmed diagnosis of OSA in the National Research Institute of Tuberculosis and Lung Disease, Masih Daneshvari Hospital. The 6-MWT was performed the day after polysomnography. The correlation between the 6-MWT and paraclinical findings during polysomnography in OSA patients was investigated.

Results: In cases with moderate to severe OSA, the male sex displayed correlation with high PCO2. Ages of patients examined displayed reversed correlation with the distance in the 6-MWT by observing the $\mathrm{O} 2$ saturation (Sat) at the end of the 6-MWT, displaying direct correlation with the duration of O2 Sat <90\% during sleep. The BMI also showed reversed correlation with the distance in the 6-MWT. Similarly, the severity of the OSA had reversed correlation with the expected distance in the test. However, patients with higher duration of O2 Sat <90\% during sleep had a higher reduction in $\mathrm{O} 2$ Sat during and after the 6-MWT. Patients with higher duration of O2 Sat $<90 \%$ during sleep also completed less overall distance in the 6-MWT ( $P$ values $<0.05$ for all).

Conclusion: It appears that the 6-MWT can be used in patients with OSA to predict severity of the desaturation in OSA beyond functional capacity. Also, it can help predict the severity of disease and assist in follow up of the OSA patients in terms of functional capacity and selection of the most appropriate treatment strategy to increase the physical ability of the patients.
\end{abstract}

\section{Background}

Obstructive Sleep Apnea (OSA) syndrome is a chronic disease affecting $9 \%$ of males and $4 \%$ of females. It most commonly affects middle-aged individuals (Al Lawati et al., 2009). It is considered a public health dilemma due to the significant association of its complications with cardiovascular morbidities and placing a major burden on health care costs (Wittmann \& Rodenstein, 2004). OSA is the most common form of sleep disorder breathing, characterized by frequent obstruction of the upper airways, causing intermittent hypoxia and consequent cardiovascular, cerebral, and metabolic complications (Pusalavidyasagar \& Iber, 2015).

\footnotetext{
* Correspondence: oldoozaloosh@yahoo.com

${ }^{2}$ Chronic Respiratory Diseases Research Center, National Research Institute of

Tuberculosis and Lung Diseases (NRITLD), Shahid Beheshti University of

Medical Sciences, Masih Daneshvari Hospital, Darabad Avenue, Shahid

Bahonar roundabout, Tehran, Iran

Full list of author information is available at the end of the article
}

The prevalence of OSA has significantly increased mainly due to the obesity epidemic worldwide.

The OSA is often associated with decreased functional status, and patients cannot tolerate physical activities. Such a limitation in physical and sport activities is related to several factors. Recurrent episodes of the upper airway obstruction, which is often accompanied by increased respiratory effort results in hypoxia and transient nocturnal hypercapnia. This leads to increases in the autonomic sympathetic activity causing arterial vasoconstriction, which is regarded as an abnormal response to exercise (Kaleth et al., 2007). Muscle dysfunction is another explanation for limited physical activity in OSA patients. Vanuxem et al. reported decreased metabolic energy of muscles in patients with OSA manifested as decreased serum level of lactate and delayed elimination of lactate from blood circulation (Vanuxem et al., 1997). On the other hand, due to strong association of OSA 
and obesity, weight gain decreases pulmonary compliance and subsequently the lung volume. This leads to increase in respiratory effort, which is another factor limiting physical activity (Alameri et al., 2010).

The Six-Minute Walk Test (6-MWT) is an affordable and simple test serving as a standard tool for assessing the physical and exercise activities of cardiovascular patients. 6-MWD(six_minute walk distance); the distance walked within 6 min is significantly correlated with maximum oxygen consumption. Moreover, the distance walked within $6 \mathrm{~min}$ is a predictor of morbidity and mortality and disease severity in patients with congestive heart failure (Cahalin et al., 1996; Roul et al., 1998), chronic obstructive pulmonary disease, pulmonary hypertension (Hoeper et al., 2000), and pulmonary interstitial diseases (Flaherty et al., 2006). In other words, the ability to walk reflects the ability to tolerate many daily activities by patients and can be regarded as an index of quality of life (Mulgrew et al., 2007; Singh et al., 2014; Holland et al., 2014).

In adults, OSA is diagnosed by polysmnography. However, this test requires over-night monitoring of patients which is costly and time consuming. Although this test can not be a substitute to the diagnostic test for obstructive sleep apnea, it can predict the patients at higher risk of significant reduction of arterial oxygen (severity of desaturation) at night can be highly valuable. If a patient who is found not to do well on the 6-MWT and history of high risk for OSA, should be referred to a sleep specialist for an overnight diagnostic sleep study, as this research study shows that most likely patients who do not do well on the 6-MWT will at least have moderate to severe obstructive sleep apnea.

Often patients are resistant to doing an overnight sleep study due to discomfort/fear of spending the night in an unknown environment. By doing a 6-MWT during the day the physician can further support concern for obstructive sleep apnea. This may convince the patient to be evaluated and treated for obstructive sleep apnea.

It seems that the 6-MWT may be one of the best candidates. In the present study, the correlation between desaturation during the 6-MWT and paraclinical findings during polysomnography in OSA patients is investigated. In other words, this study aimed to show the severity of OSA had reverse correlation with expected distance in moderate and severe OSA. The effect of nocturnal intermittent hypoxia (despite compensation) on physical activity of individuals.

\section{Methods}

\section{Study design}

This cross-sectional study was conducted on 47 patients with confirmed diagnosis of OSA in the National Research Institute of Tuberculosis and Lung Disease
(NRITLD), Masih Daneshvari Hospital (a referral hospital in Tehran, Iran) during 1 year period starting from August 2012 to June 2013. Written informed consent was obtained from all of the patients prior to their participation in the study. The study protocol was approved by the ethics committee of the Shahid Beheshti University of Medical Sciences and local scientific committees and it was conducted in compliance with the Declaration of Helsinki.

\section{Eligibility}

All patients were newly diagnosed with moderate or severe OSA (aged $<80$ years, BMI $<35 \mathrm{~kg} / \mathrm{m}^{2}$, height $<2 \mathrm{~m}$ ). In addition, they had normal chest radiographs and spirometry results. The patients had no history of using continuous positive airway pressure (CPAP). The exclusion criteria were including: psychosomatic disorders, acute arthritis, concomitant pulmonary diseases, congestive heart failure, ischemic heart disease, neuromuscular disorders, bronchospasm, severely decreased $\mathrm{O}_{2}$ saturation, contraindications for the 6-MWT [including unstable angina symptoms during the month preceding the test, resting heart rate $>120 \mathrm{bpm}$, systolic-blood-pressure (SBP) > $180 \mathrm{mmHg}$ and/or diastolic-blood-pressure (DBP) $\geq 100 \mathrm{mmHg}$ ], chronic obstructive pulmonary disease and other pulmonary diseases.

\section{Polysomnography}

Diagnosis of OSA and its severity is determined based on the Apnea-Hypopnea Index (AHI) score suggested by the American Academy of Sleep Medicine guidelines (Richard et al., 2015). The degree of hypoxemia was also determined by a sleep study in the Sleep Disorders Center. The polysomnography consisted of an overnight study and video monitoring,Standard EEG by AASM protocol,muscle tone and leg movements by chin and leg electromyography, eye movements by electrooculography, heart rate by electrocardiography, and oxygen saturation by pulse oximetry. Thoracic and abdominal belts were used for chest and abdominal wall movements.

Snoring was assessed using a microphone. The polysomnogram was analyzed manually using AASM guideline with Philips Respironic (Alice) Device. The Desaturation Index (DI) is defined as the number of desaturation events per hour of sleep. The severity of OSA was then graded based on AHI in accordance with the American Academy of Sleep Medicine guidelines (Richard et al., 2015)as mild (5-15 AHI events/h), moderate (15-30 AHI events/h), or severe (>30 AHI events/h).

Obstructive apnea is defined as absence of airflow for more than $10 \mathrm{~s}$ in presence of continued respiratory effort. Hypopnea here is defined as a reduction in airflow by $\geq 30 \%$, compared with baseline that lasted for more than $10 \mathrm{~s}$, resulting in 3\% decrease in oxygen saturation, 
or arousal. The AHI score is defined as the number of apnea and hypopnea episodes per hour of sleep and is calculated for the total sleep time. The OSA is defined according to the International Classification of Sleep Disorders (International Classification of Sleep Disorders, 2005).

\section{The 6-MWT}

The 6-MWT was performed the day after polysomnography. It was conducted outdoors along a seldomtraveled, flat, straight hospital corridor (40 m long) and between eight and $10 \mathrm{AM}$ according to the American Thoracic Society (ATS) protocol (ATS statement, 2002). Subjects avoided vigorous exercise for $2 \mathrm{~h}$ prior to the test and advised to have light meal and wear comfortable clothing and appropriate shoes. $10 \mathrm{~min}$ before the onset of the 6-MWT, resting dyspnea, heart rate, and $\mathrm{O}_{2}$-saturation were measured. All the tests were performed by the same technician trained according to the ATS guidelines.

At the end of the 6-MWT, the same data in addition to the distance walked during the 6-MWT (6-MWD) and the number of stops during the 6-MWT, were recorded. Also, the patients were asked to rate their level of perceived dyspnea (breathless) using the Borg scale (see Additional file 1).

A 6-MWT result below the lower limit of normal was considered as "clinically significant" and showed walk intolerance. Other 6-MWT definitions were as follows:

- Stopping during the 6-MWT was defined as a sign of walk intolerance.

- The difference in oxygen saturation $>4$ points before and after the test, was regarded as "clinically significant desaturation".

- Any dyspnea at the end of the test scored between 5 to 10 (according to the Borg scale) was considered as "clinically significant" and regarded as walk intolerance.

- If the heart rate at the end of the 6-MWT was $<60 \%$ compared to the baseline then it was regarded as chronotropic incompetence (Ben Saad et al., 2014).

\section{Statistical analysis}

Sample size was calculated according to the eq. (1):

$$
n=z 2 \sigma 2 / d 2
$$

where $d=0.3 \sigma$ with $5 \%$ level of significance for alpha. The sample size was calculated to be 43 as such. To assess the differences in categorical variables between the two groups, the chi-square test or Fisher's exact test was used. The differences in quantitative variables between the two groups were evaluated using the Student's t-test,
ANOVA or non-parametric Mann-Whitney test. Passive smoking was referred to cigarette smoke breathed in by non-smokers from active smokers. Ex-smoker (or former smoker) was defined as a person who reported not smoking at one examination while he/she had reported smoking at the examination 4 years earlier (i.e., individuals who had quit for $\leq 4$ years or not smoking for 2 or more consecutive examinations after an examination at which they had reported smoking). Non-smoker was defined as an individual who had smoked less than 100 cigarettes in his/her lifetime (Sari et al., 2016). Statistical analysis was performed using SPSS version 18 (SPSS Inc.; Chicago, IL, USA).

\section{Results}

Of 47 eligible patients, 33 (70.21\%) were males and 14 (29.79\%) were females. Male/Female ratio was $2 / 3$. Demographic characteristics of the patients are summarized in Table 1.

Polysomnographic findings are presented in Table 2 . Severe and moderate OSA were seen in $23.4 \%(n=11)$ and $75.6 \%(n=36)$ of patients, respectively.

Univariate analysis between age, sex, smoking status, and other variables and polysomnographic data revealed a significant correlation between age and DI $(r=0.456$, $P=0.001)$.

Among the 6-MWT parameters in patients with OSA Only significant raise of heart beat before and after of 6MWT ( $\mathrm{P}=<0.001)$ was determined.

Table 3 presents differences between patients with moderate versus severe OSA after the 6-MWT. The 6MWD in the severe OSA group $(477.44 \pm 275.65 \mathrm{~m})$ was statistically similar $(P=0.173)$ to that in the moderate OSA group $(555.45 \pm 107.82 \mathrm{~m})$. However, the percentage predicted by the 6-MWT for the severe OSA group $(75.91 \pm 36.45)$ was significantly shorter than that for the moderate group $(92.4 \pm 10.41 \mathrm{~m}, P=0.020)$.

Table 1 Characteristics of the study group

\begin{tabular}{|c|c|}
\hline \multicolumn{2}{|c|}{ Variable mean $\pm S D^{a}$ (range) } \\
\hline Age (years) & $54.4 \pm 14.33(22-80)$ \\
\hline Height $(\mathrm{cm})^{\mathrm{b}}$ & $165.7 \pm 8.77(147-182)$ \\
\hline Weight $(\mathrm{Kg})^{c}$ & $91.9 \pm 15.58(58-125)$ \\
\hline $\mathrm{BMI}^{\mathrm{d}}\left(\mathrm{Kg} / \mathrm{m}^{2}\right)^{\mathrm{e}}$ & $33.74 \pm 6.23(22.5-50)$ \\
\hline \multicolumn{2}{|l|}{ Smoking status } \\
\hline Active smoker & $8.5 \%(n=4)$ \\
\hline Passive smoker & $6.4 \%(n=3)$ \\
\hline Ex-smoker & $17 \%(n=8)$ \\
\hline Non-smoker & $68.1 \%(n=32)$ \\
\hline
\end{tabular}

Abbreviations: a SD: Standard deviation, $b \mathrm{~cm}$ : Centimeter, c kg: Kilograms, $d$ BMI: Body mass index,

e $\mathrm{kg} / \mathrm{m}^{2}:$ Kilograms per square meter 
Table 2 Polysomnographic data of the patients with OSA

\begin{tabular}{lllll}
\hline Variable mean $\pm \mathrm{SD}^{\mathrm{a}}$ & Male & Female & Total & $P$ value \\
\hline $\mathrm{AHI}^{\mathrm{b}}$ & $53.27 \pm 29.62$ & $47.42 \pm 18.78$ & $51.53 \pm 26.78$ & 0.422 \\
Desaturation index(DI) & $56.02 \pm 25.95$ & $63.9 \pm 26.71$ & $55.39 \pm 25.91$ & 0.804 \\
$\mathrm{PaCO}_{2}^{\mathrm{c}}$ & $51.28 \pm 5.35$ & $46.46 \pm 7.53$ & $49.72 \pm 6.46$ & $0.025^{*}$ \\
Time spent $<90 \%{ }^{\mathrm{d}}$ & $110.21 \pm 97.25$ & $138.95 \pm 98.81$ & $118.77 \pm 97.54$ & 0.391 \\
Severe OSA & $24(72.7 \%)$ & $12(85.7 \%)$ & $36(76.7 \%)$ & 0.464 \\
Moderate OSA & $9(27.3 \%)$ & $2(14.3 \%)$ & $11(23.4 \%)$ & \\
\hline
\end{tabular}

Abbreviations: a SD: Standard deviation, b AHI: Apnea hypopnea index, c PaCo 2 : Partial pressure of carbon dioxide in atrial blood, $d$ Time spent < 90\% (\%): Time (minute) spent during sleep with $\mathrm{O}_{2}$ saturation < 90\%, OSA: Obstructive sleep apnea

* Significant $P$ value

For further evaluation, the association of each demographic and polysomnographic parameter with the 6MWT results were examined. The distance walked by the OSA patients negatively correlated with age $(r=-0.391, P=0.0130)$ and time spent $<90 \%$ ( $r=-0.316 P=0.031)$ in polysomnographic test and positively with the BMI $(r=0.516, P<0.001)$. In addition, BMI had a reverse correlation with $\mathrm{SaO}_{2}$ at the end of the $6 \mathrm{MWT}(r=-0.0456, P=0.001)$. However, the 6-MWD did not correlate with sex, smoking status, or other polysomnographic variables. In addition, polysomnographic data with the 6-MWT results were correlated. There was a significant reverse correlation between time spent $<90 \%$ and AHI during polysomnography with $\mathrm{SaO}_{2} \%$ at the end of the 6-MWT $(r=-0.521, P<0.001$ and $r=-0.312, P=0.044$ ). Furthermore, there was a significant correlation between the time spent $<90 \%$ during polysomnography and DI during the $6-\mathrm{MWT}(r=-0$. 328, $P=0.013$, Fig. 1 ).

\section{Discussion}

To the best of the authors' knowledge, the current study is among the very few studies on OSA patients in Iran and in the Middle East. Affliction with OSA is associated with decreased functional capacity and drop in daily physical activities. Such disabilities increase with age and BMI. Drop in physical activities can adversely affect the general health and quality of life of the patients. A significant association was found between the percentage of the 6-MWD and the severity of OSA in the current study. This highlights the significance of early detection of the OSA patients and indicates the impact of early diagnosis on maintenance of physical activity in such patients. On the other hand, the significant association between the 6-MWD and polysomnographic parameters such as time spent $<90 \%$ indicates the optimal efficacy of this test (which is simpler than polysomnography) for detection of patients at risk of nocturnal hypoxia. The role of nocturnal intermittent hypoxia in disability of patients and also as a possible cause of dyspnea can also be evaluated as such.

A previous study has reported an association between sex and the 6-MWD and showed that female patients with OSA walked a significantly shorter distance (Pływaczewski et al., 2008). However, in the current study, no association was found between sex and any of the 6-MWT or polysomnographic findings except for $\mathrm{PaCO}_{2}$, which was significantly higher in males in polysomnography. Higher prevalence of OSA in males and higher $\mathrm{PaCO}_{2}$ may indicate potentially higher susceptibility of males to develop OSA and that slight changes in $\mathrm{PaCO}_{2}$ may cause significant changes in $\mathrm{SaO}_{2}$ during sleep. Studies on the effect of sex on physical activity tolerance are scarce and further investigations are required in this respect.

Most of the patients with OSA are in mid-life age (Jennum \& Riha, 2009), which may be due to several factors such as structural changes and fat deposition in the para-pharyngeal area and elongation of soft palate.

Table 3 6-MWT parameters in patients with moderate vs severe OSA

\begin{tabular}{llll}
\hline & Moderate OSA ${ }^{\text {a }}$ group & Severe OSA group & $P$ value \\
\hline $6-M_{W D}{ }^{b}, m^{c} \pm \mathrm{SD}^{\mathrm{d}}$ & $555.45 \pm 107.82$ & $477.44 \pm 275.65$ & 0.173 \\
$6-\mathrm{MWD}(\%)^{\mathrm{f}} \pm \mathrm{SD}$ & $92.4 \% \pm 10.41$ & $75.91 \pm 36.45$ & 0.020 \\
$\mathrm{HR}{ }^{\mathrm{g}}$, beats/min(from base-line) $\pm \mathrm{SD}$ & $30.09 \pm 16.86$ & $27.83 \pm 19.26$ & 0.733 \\
$\mathrm{HR}$ 'beats/min after 6-MWT $\pm \mathrm{SD}$ & $101.27 \pm 24.31$ & $104.45 \pm 29.88$ & 0.753 \\
$\mathrm{SaO}_{2} \%{ }^{\mathrm{h}}$ at end of 6-MWT $\pm \mathrm{SD}$ & $91 \pm 4.97$ & $90.22 \pm 7.67$ & 0.758 \\
$\mathrm{SaO}_{2} \%$ at end of 6-MWT from base line $\pm \mathrm{SD}$ & $2.09 \pm 5.26$ & $1 \pm 5.99$ & 0.596 \\
\hline
\end{tabular}

Abbreviations: a OSA: obstructive sleep apnea, b 6-MWD: distance walked during the 6-MWT, c 6-MWD, $m$ : is expressed in absolute (meter), d SD: Standard deviation, $f$ 6MWD: 6-MWD is as percentage predicted (\%) values, $g$ HR: Heart rate, $h \mathrm{SaO}_{2}$ : Oxygen saturation 


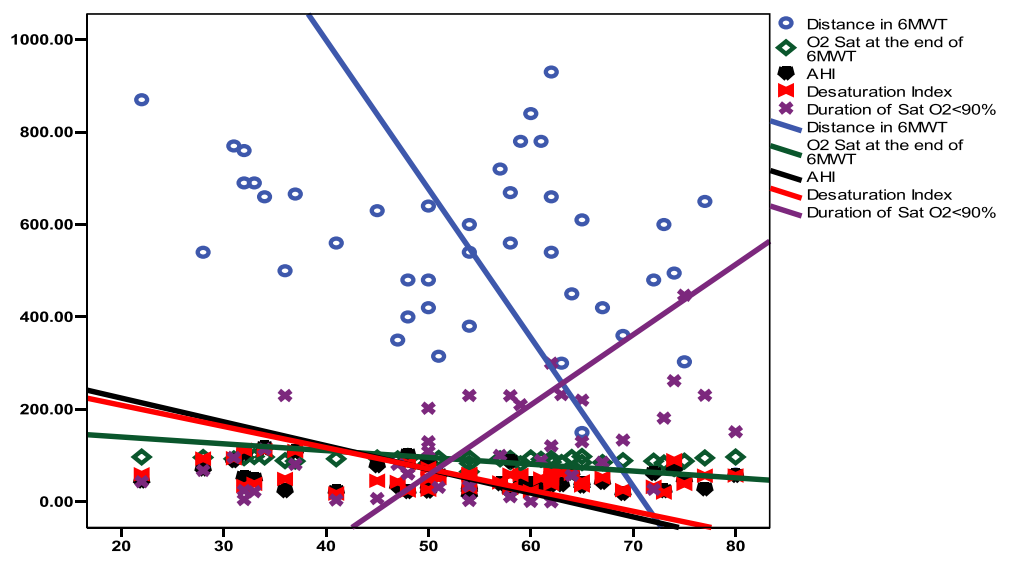

Fig. 1 Correlation of time spent $<90 \%, \mathrm{AHI}$ and DI during polysomnography with distance walked and $\mathrm{O} 2$ saturation in the 6-min walk test

These changes probably result in upper airway obstruction in the elderly (Eikermann et al., 2007). In the present study, an inverse correlation was found between age and the 6-MWD and oxygen saturation rate upon the completion of the test. On the other hand, age had a significant association with AHI and DI during polysomnography, indicating the possibility of nocturnal oxygen reduction and drop in daily physical activity with age. Thus, it seems that the 6-MWT can be highly efficient and applicable for detection of the elderly who carry the risk of OSA after rolling out other decreasing causes of capacity, but have yet to manifest its typical signs and symptoms.

Some researchers reported that is not a significant difference in dyspnea upon completion of the 6-MWT between those with OSA and healthy adults (Kaleth et al., 2007). However, this is a controversial topic since some other studies have demonstrated higher frequency of dyspnea upon completion of the 6-MWT in patients with OSA compared to others (Alameri et al., 2010). In the current study, approximately $80 \%$ of the patients had dyspnea upon completion of the 6-MWT; out of which, $30 \%$ had abnormal results with regard to the assessed parameters. This value was higher than that in studies reported by Pływaczewski et al. (18.5\%) (Pływaczewski et al., 2008) and Ben Saad et al. (13\%) (Ben Saad et al., 2015). Such a difference in the results of the studies may be due to the fact that Pływaczewski et al. (Pływaczewski et al., 2008) included patients with variable degrees of OSA, which can obviously affect the results. In the study by Ben Saad et al. (Ben Saad et al., 2015) patients were under treatment with CPAP; while the CPAP patients were excluded in the present study. Sample size and male/female ratio can also be responsible for the variability in the results of studies.

The 6-MWD in patients with severe OSA was significantly less than that in other patients, demonstrating the significant effect of disease severity on reduction of daily activities. It also shows that the patients who walk a shorter distance in the 6-MWT probably experience nocturnal apnea and hypoxia, which may be related to disease severity.

High BMI and obesity decrease functional capacity due to increased workload and decreased respiratory function and significantly affect the ability to perform physical activities. In the current study, higher BMI was associated with shorter 6-MWD, which is in agreement with the results of previous studies (Kaleth et al., 2007; Alameri et al., 2010; Pływaczewski et al., 2008). This finding suggests that weight control programs focusing on a healthy diet and regular exercise can significantly improve the performance of the patients.

Adults commonly experience a reduction in blood pressure and heart rate during sleep, which is probably due to the decreased activity of the autonomous system (sympathetic and parasympathetic) (Alameri et al., 2010). Interestingly, the opposite occurs in the OSA patients, and a significant increase in sympathetic tone during the nocturnal arousal episodes results in a significant increase in heart rate during sleep (Narkiewicz et al., 1998; Somers et al., 1993). The pattern of the heart rate response in the OSA patients is still a matter of controversy. Alameri et al. (Alameri et al., 2010) showed that, compared to healthy individuals, acceleration of heart rate is slower in the OSA patients and it takes longer for the heart rate to return back to normal after exercise and physical activity. In the current study, a significant increase in heart rate was noted after the conduction of the 6-MWT compared to baseline, which indicates the probable role of autonomous system dysregulation in the pathogenesis of OSA.

Furthermore, DI during the 6-MWT can provide us with valuable information about the desaturation caused by exercise and severity of the disease. The significant association of DI during the 6-MWT and time spent $<90 \%$ during polysomnographymay be attributed 
to the fact that decreased oxygen during sleep in patients with OSA can alter oxygen availability through alveolar capillaries in such a way to limit physical activities. Tachycardia or other arrhythmia during nocturnal hypoxemia can result in pulmonary arterial hypertension. These events can also explain nocturnal desaturation (Shepard et al., 1985; Coccagna \& Lugaresi, 1978). By considering the fact that the reduction in arterial oxygen is correlated with the severity of the OSA, abnormal DI upon completion of the 6-MWT was expected in our patients, since they had moderate to severe OSA. The same results were reported in previous studies (Alameri et al., 2010; Ben Saad et al., 2015; Shepard et al., 1985; Coccagna \& Lugaresi, 1978). This is particularly important as DI at the end of the 6-MWT can predict the level of nocturnal oxygen reduction in patients with OSA. This is useful in treatment planning and selection of treatment strategies. Casanova et al. demonstrated that in patients with chronic obstructive pulmonary disease, DI during the 6-MWT negatively affected the prognosis of patients. However, the accuracy of this finding in the OSA patients has not been yet tested (Casanova et al., 2008).

Significant correlation of arterial oxygen saturation, DI and AHI at the end of the 6-MWT with time spent $<90 \%$ during polysomnography indicates that patients who have lower arterial oxygen saturation rate during daily activities experience greater and more significant changes in oxygenation at night. This further highlights the significance of the 6-MWT as a simple and efficient tool for assessment of the OSA patients. The 6-MWT is generally a screening tool for cardiac function and lower airway disease, but this study has shown that it can also be used to assess for an upper airway disease (i.e obstructive sleep apnea).

The present study had some limitations: First, there is not a control group. If a control group was present, possible significant differences between the test and control groups could have further confirmed the efficacy of the 6-MWT for early detection of patients with OSA or those at risk of developing OSA. The second limitation is that other factors limiting physical activities and subsequently the test results (such as muscle strength and psychological factors) were not taken into account.

\section{Conclusion}

In general, based on the above-mentioned findings, it appears that the 6-MWT can be used in patients with OSA to assess their physical ability and tolerance of activities. Also, it can help predict the severity of the disease and assist in selection of the most appropriate treatment strategy to increase the physical ability of the patients.

\section{Additional file}

Additional file 1: Borg rating perceived exertion scale. (DOCX 13 kb)

\author{
Acknowledgements \\ Not applicable. \\ Funding \\ There was no any providing funding. Authors paid all expenses including \\ data collection, analysis and also writing by themselves.
}

\section{Availability of data and materials}

The datasets generated during and/or analyzed during the current study are available (in sleep laboratory data box) from the corresponding author on reasonable request.

\section{Authors' contributions}

Study conception and designs: PAN and MM had the main rule in this part Acquisition of data: HAT collected data specially 6- MWT datasets, PAN and MM gathered PSGs' results and PAN also reported the PSG tests. Analysis and interpretation of data: Analysis and conclusion of data was done by OA and HAT. Drafting of manuscript: HAT and OA prepared initial manuscript. Critical revesion: PAN and OA wrote critical review. All authors reviewed the final article and approved the work.

\section{Ethics approval and consent to participate}

To whom it may concern. This is to certify that research project entitled "Can 6-minute walk test predict severity of obstructive sleep apnea?" referred to Masih Daneshavri Hospital is carried out under supervision of Dr. Parisa Adimi as the Principal Investigator. The project registration number is 786 dated February 2, 2012. The research project was also approved by the ethics committee in September 13, 2011. Dr. Parisa Farnia. Deputy Head of Research. National Research Institute for Tuberculosis and Lung Diseases (NRITLD), Masih Daneshvari Hospital, Darabad, Niavaran Tehran, Iran.

\section{Consent for publication \\ Not applicable.}

\section{Competing interests}

The authors declare that they have no competing interests

\section{Publisher's Note}

Springer Nature remains neutral with regard to jurisdictional claims in published maps and institutional affiliations.

\section{Author details \\ ${ }^{1}$ Clinical Tuberculosis and Epidemiology Research Center, National Research Institute of Tuberculosis and Lung Diseases (NRITLD), Shahid Beheshti University of Medical Sciences, Tehran, Iran. ${ }^{2}$ Chronic Respiratory Diseases Research Center, National Research Institute of Tuberculosis and Lung Diseases (NRITLD), Shahid Beheshti University of Medical Sciences, Masih Daneshvari Hospital, Darabad Avenue, Shahid Bahonar roundabout, Tehran, Iran. ${ }^{3}$ Tracheal Diseases Research Center, National Research Institute of Tuberculosis and Lung Diseases (NRITLD), Shahid Beheshti University of Medical Sciences, Tehran, Iran.}

Received: 2 May 2017 Accepted: 16 August 2017

Published online: 05 September 2017

\section{References}

Al Lawati NM, Patel SR, Ayas NT. Epidemiology, risk factors, and consequences of obstructive sleep Apnea and short sleep duration. Prog Cardiovasc Dis. 2009; 51(4):285-93.

Alameri H, Al-Kabab Y, BaHammam A. Submaximal exercise in patients with severe obstructive sleep apnea. Sleep and Breathing. 2010;14(2):145-51.

ATS statement. Guidelines for the six-minute walk test. Am J Respir Crit Care Med. 2002;166:111e7. 
Ben Saad H, Babba M, Boukamcha R, et al. Investigation of exclusive narghile smokers: deficiency and incapacity measured by spirometry and 6-minute walk test. Respir Care. 2014;59:1696e709.

Ben Saad H, Ben Hassen K, Ghannouchi I, Latiri I, Rouatbi S. 6-min walk-test data in severe obstructivesleep-apnea-hypopnea-syndrome (OSAHS) under continuous-positive-airway-pressure (CPAP) treatment. Respir Med. 2015;109; 642-55.

Cahalin LP, Mathier MA, Semigran MJ, Dec GW. DiSalvoTG. The six-minute walk test predicts peak oxygen uptake and survival in patients with advanced heart failure. Chest. 1996;110(2):325-32.

Casanova C, Cote C, Marin JM, et al. Distance and oxygen desaturation during the 6-min walk test as predictors of long-term mortality in patients with COPD. Chest. 2008;134:746-5.

Coccagna G, Lugaresi E. Arterial blood gases and pulmonary and systemic arterial pressure during sleep in chronic obstructive pulmonary disease. Sleep. 1978; 1:117-24

Eikermann M, Jordan AS, Chamberlin NL, et al. The influence of aging on pharyngeal collapsibility during sleep. Chest. 2007;131:1702-9.

Flaherty KR, Andrei AC, Murray S, Fraley C, Colby TV, Travis WD, et al. Idiopathic pulmonary fibrosis: prognostic value of changes in physiology and six minute hall walk. Am J Respir Crit Care Med. 2006;174(7):803-9.

Hoeper MM, Schwarze M, Ehlerding S, Adler-Schuermeyer A, Spiekerkoetter E, Niedermeyer J, et al. Long-term treatment of primary pulmonary hypertension with aerosolized iloprost, a prostacyclin analogue. N Engl J Med. 2000;342(25):1866-70.

Holland AE, Spruit MA, Troosters T, et al. An official European Respiratory Society/ American Thoracic Society technical standard: field walking tests in chronic respiratory disease. Eur Respir J. 2014;44:1428-46.

International Classification of Sleep Disorders. Diagnostic \& coding manual. 2nd ed. Westchester: American Academy of Sleep Medicine; 2005.

Jennum P, Riha RL. Epidemiology of sleep apnoea/hypopnoea syndrome and sleep-disordered breathing. Eur Respir J. 2009;33:907-14.

Kaleth AS, Chittenden TW, Hawkins BJ, Hargens TA, Guill SG, Zedalis D, et al. Unique cardiopulmonary exercise test responses in overweight middle-aged adults with obstructive sleep apnea. Sleep Med. 2007:8(2):160-8.

Mulgrew T, Ryan CF, Fleetham JA, et al. The impact of obstructive sleep apnea and daytime sleepiness on work limitation. Sleep Med. 2007:9:42-53.

Narkiewicz K, van de Borne PJ, Cooley RL, Dyken ME, Somers VK. Sympathetic activity in obese subjects with and without obstructive sleep apnea. Circulation. 1998;98(8):772-6.

Pływaczewski R, Stokłosa A, Bieleń P, Bednarek M, Czerniawska J, Jonczak L, Górecka D. Sliwiński six-minute walk test in obstructive sleep apnoea. Pneumonol Alergol Pol. 2008;76(2):75-82.

Pusalavidyasagar S, Iber C. Current medical concepts in obstructive sleep apnea. Oper Tech Otolaryngol Head Neck Surg. 2015;26(2):52-8

Richard B, Charlene E, Susan M, Robin M, Carole L and Bradley V. The American Academy of sleep medicine the AASM manual for the scoring of sleep and associated events: rules, terminology and technical specifications,version 2. 2(2015).

Roul G, Germain P, Bareiss P. Does the 6-minute walk test predicts the prognosis in patients with NYHA class II or III chronic heart failure? Am Heart J. 1998; 136(3):449-57.

Sari AA, Rezaei S, Arab M, Majdzadeh R, Matin BK, Zandian H. Effects of smoking on cost of hospitalization and length of stay among patients with lung cancer in Iran: a hospital-based study. Asian Pac J Cancer Prev. 2016;17(9): $4421-6$.

Shepard JW Jr, Garrison MW, Grither DA, Evans R, Schweitzer PK. Relationship of ventricular ectopy to nocturnal oxygen desaturation in patients with chronic obstructive pulmonary disease. Am J Med. 1985;78:28-34.

Singh SJ, Puhan MA, Andrianopoulos V, et al. An official systematic review of the European Respiratory Society/American Thoracic Society: measurement properties of field walking tests in chronic respiratory disease. Eur Respir J. 2014:44:1447-78.

Somers VK, Dyken ME, Mark AL, Abboud FM. Sympathetic-nerve activity during sleep in normal subjects. N Engl J Med. 1993;328(5):303-7.

Vanuxem D, Badier M, Guillot C, Delpierre S, Jahjah F, Vanuxem P. Impairment of muscle energy metabolism in patients with sleep apnoea syndrome. Respir Med. 1997;91(9):551-7.

Wittmann V, Rodenstein DO. Health care costs and the sleep apnea syndrome. Sleep Med Rev. 2004:8:269e79.

\section{Submit your next manuscript to BioMed Central and we will help you at every step:}

- We accept pre-submission inquiries

- Our selector tool helps you to find the most relevant journal

- We provide round the clock customer support

- Convenient online submission

- Thorough peer review

- Inclusion in PubMed and all major indexing services

- Maximum visibility for your research

Submit your manuscript at www.biomedcentral.com/submit
Biomed Central 